\title{
SPARC expression is negatively correlated with clinicopathological factors of gastric cancer and inhibits malignancy of gastric cancer cells
}

\author{
JUNLING ZHANG ${ }^{1}$, PENGYUAN WANG ${ }^{1}$, JING ZHU $^{1}$, WEI WANG ${ }^{1}$, JIE YIN $^{1}$, CHI ZHANG $^{2}$, \\ ZIYI CHEN ${ }^{1}$, LIE SUN ${ }^{1}$, YUANLIAN WAN ${ }^{1}$, XIN WANG $^{1}$, GUOWEI CHEN $^{1}$ and YUCUN LIU ${ }^{1}$ \\ ${ }^{1}$ Department of General Surgery, Peking University First Hospital, Beijing 100034; ${ }^{2}$ Department of General Surgery, \\ Provincial Hospital Affiliated to Shandong University, Jinan, Shandong 250021, P.R. China
}

Received January 29, 2014; Accepted March 4, 2014

DOI: 10.3892/or.2014.3118

\begin{abstract}
Secreted protein acidic and rich in cysteine (SPARC) is a glycoprotein which plays multiple roles in different types of cancer. Our previous study showed that SPARC overexpression inhibited the growth and angiogenesis of tumors, and reduced expression of vascular endothelial growth factor (VEGF). However, the relationship between SPARC expression and clinicopathological factors of gastric cancer (GC) is controversial, and the role of SPARC in GC remains unclear. We evaluated expression of SPARC in 65 human GC tissues using immunohistochemistry (IHC). The results indicated that SPARC expression was negatively correlated with clinicopathological factors of GC. In vitro assay showed that SPARC overexpression decreased proliferation and clonogenicity by suppressing CD44 expression. In addition, SPARC overexpression inhibited VEGF induced proliferation and arrested cell cycle of GC cells by reducing the activation of VEGFR2, ERK1/2 and AKT signaling pathways. SPARC suppressed the invasion and migration of GC by reducing MMP-7, MMP-9, $\mathrm{N}$-cadherin, $\mathrm{Sp} 1$ and $\mathrm{p}$-ERK1/2 expression. In the in vivo assay, cancer metastasis mouse models were established by tail vein injection. The results revealed that the lung metastases of SPARC-overexpressing GC cells in the mice were much fewer than those of control cells.
\end{abstract}

\section{Introduction}

Gastric cancer (GC) has become a significant health problem globally, with a total of 989,600 new cases and 738,000 deaths

Correspondence to: Professor Guowei Chen or Professor Yucun Liu, Department of General Surgery, Peking University First Hospital, 8 Xishiku Street, Xicheng, Beijing 100034, P.R. China

E-mail: guoweichen@263.net

E-mail: yucunliu@bjmu.edu.cn

Key words: SPARC, VEGFR2, proliferation, clonogenicity, invasion, gastric cancer estimated to have occurred in 2008, accounting for $8 \%$ of the total cases and $10 \%$ of all cancer-related deaths, respectively (1). The geographical distribution of GC exhibits wide international variation and $>70 \%$ of new cases and deaths occur in developing countries, including $42 \%$ in China (2).

Secreted protein acidic and rich in cysteine (SPARC) is a multi-faceted secreted glycoprotein, which is abnormally expressed by different types of cancer. SPARC is silenced in many types of cancer cells, but stromal fibroblasts adjacent to the tumors frequently express SPARC. The role of SPARC is complicated and appears to depend on diverse given microenvironments. In certain types of cancer, such as melanoma, SPARC is associated with a highly aggressive tumor phenotype. However, SPARC seemed to function as a tumor suppressor, since hyper-methylation and loss of SPARC gene expression had been detected in lung, ovarian, pancreatic, colorectal and breast cancer (3). Colon cancer patients with low or absent expressing SPARC had significantly poorer overall and disease-free survival. SPARC expression was significantly different in colon cancers with lymph node metastasis and differentiation degree of tumor (4). Moreover, the clonogenic and migratory capabilities were largely decreased in SPARCoverexpressing hepatocellular carcinoma cells (5). Our previous studies showed that endogenous SPARC inhibited the malignant phenotype in pancreatic cancer (6). In GC, we found endogenous SPARC inhibited the angiogenesis by suppressing expression of vascular endothelial growth factor (VEGF) and MMP-7 (7). Increased SPARC expression is associated with a better prognosis of these tumors $(3,8,9)$. Previous studies showed that SPARC antagonized the effect of bFGF on the migration of aortic endothelial cells (10), and inhibited the proliferation and migration of aortic endothelial cells (11). Mechanisms of SPARC action were also examined in SPARC wild-type and SPARC knockout mice injected with ovarian cancer cells. Wild-type mice had lower levels of matrix metalloproteinases (MMPs) and VEGF (12).

Acquisition of metastatic phenotype of cancer cells consists of multiple steps including epithelial mesenchymal transition (EMT). Changes in cadherin expression patterns may play a role in the process of EMT and cellular motility. Non-epithelial cadherin, including $\mathrm{N}$-cadherin, was found to 
induce a mesenchymal-scattered phenotype associated with reduced E- cadherin in tumors. The MMP family of enzymes contributes to both normal and pathological tissue remodeling. It is believed that MMP expression is highly correlated with invasion, metastasis and angiogenesis of GC.

Previous immunohistochemistry (IHC) studies showed that normal gastric mucosa tissues expressed low level of SPARC. In GC tissues, SPARC expression disappeared in cancer cells but localized in stromal cells surrounding the cancer $(13,14)$. However, the relationship between SPARC expression and clinicopathological factors of GCs is controversial. Therefore, in the present study, we examined the correlation between SPARC and clinicopathological factors. Secondly, we assessed the effect of SPARC overexpression on the growth and clonogenicity of GC cells. In addition, the purpose of the present study was to investigate whether SPARC overexpression is involved in the process of metastasis via EMT.

\section{Materials and methods}

Antibodies and reagents. Antibodies against SPARC (Cell Signaling Technology, Danvers, MA, USA) were used for western blotting (WB) and IHC. E-cadherin, N-cadherin, Sp1, (p-)ERK1/2, p21, cyclin-D1 (Cell Signaling Technology), (p)-VEGFR2 (Abcam, Cambridge, MA, USA) were used for WB. CD44 (Cell Signaling Technology) was used in flow cytometry (FCM). All other reagents were of analytical grade or better.

Surgical samples. Between 2004 and 2008, a total of 65 patients with GC participated in this study. Fresh samples of both tumor tissue and adjacent normal mucosa were obtained and fixed in formalin immediately after surgery. All the noncancerous tissues were obtained at a distance of $>5 \mathrm{~cm}$ from GCs. The research was approved by the Institutional Review Board of Peking University First Hospital and informed consent was obtained from all patients.

Immunohistochemistry. Sections $(4 \mu \mathrm{m})$ from the paraffinembedded,formalin-fixed GC tissues were fixed on the charged slides for immunohistochemical analysis using non-biotin detection system (GTVision III Anti-Mouse/Rabbit-HRP; Gene Tech). Primary rabbit monoclonal antibodies to SPARC (1:200) were used in the present study. All slides were deparaffinized with xylene and rehydrated through graded concentrations of ethanol ending with distilled water. Then, endogenous peroxidase was blocked by $0.3 \% \mathrm{H}_{2} \mathrm{O}_{2}$ for $30 \mathrm{~min}$. Sections for SPARC were subjected to microwave antigen retrieval with $0.1 \mathrm{M}$ EDTA buffer $\left(\mathrm{pH} \mathrm{8.0)}\right.$ at $95^{\circ} \mathrm{C}$ for $15 \mathrm{~min}$, and were then incubated with primary antibodies overnight at $4^{\circ} \mathrm{C}$, followed by GTVision detection incubated for $30 \mathrm{~min}$ at $37^{\circ} \mathrm{C}$. The staining was visualized by incubating with DAB for $5 \mathrm{~min}$, then counter-stained with hematoxylin. Sections of known positive specimens were used as positive controls. Sections incubated with phosphate-buffered saline (PBS) instead of primary antibody were used as negative controls. The intensity of immunostaining for SPARC was reviewed and scored according to the location of cytoplasm. The results were presented by two independent pathologists without knowledge of the clinicopathological parameters of the patients. The proportion of cells with SPARC expression was rated as follows: 0 point, $\leq 5 \%$ positive cells; 1 point, 6-25\% positive cells; 2 points, $26-50 \%$ positive cells; 3 points, $\geq 51 \%$ positive cells. The intensity of staining varied from weak to strong. The intensity was classified as a scale of 0 (no staining); 1 (weak staining, light yellow); 2 (moderate staining, yellowish brown) and 3 (strong staining, brown). Staining index was calculated as the product of staining intensity score and the proportion of positive cells. We obtained the staining index with scores of $0,1,2,3,4,6$ or 9; a staining index score $\geq 4$ was used to define cells with high SPARC expression, and a staining index score $\leq 3$ was used to indicate low SPARC expression (14).

Cell culture. Human GC cell lines BGC-823, SGC-7901 were obtained from the Cancer Institute of the Chinese Academy of Medical Science. BGC-P (parental BGC-823), SGC-P (parental SGC-7901) cells were grown in complete RPMI-1640. BGC-EV (transfected with empty vector), BGC-SP (overexpressing SPARC cDNA); SGC-EV (transfected with empty vector) and SGC-SP (overexpressing SPARC cDNA) cells were grown in complete RPMI-1640 with G418 $(50 \mu \mathrm{g} / \mathrm{ml})$. All cells were maintained in monolayer cultures at $37^{\circ} \mathrm{C}$ in humidified air with $5 \% \mathrm{CO}_{2}$.

Establishment of BGC-SP, SGC-SP clones. Briefly, 150,000 BGC-823 or SGC-7901 cells were plated per well in a 6-well plate and allowed to attach overnight. Equimolar amounts of pcDNA3.1 with full length SPARC cDNA vector or the empty vector were incubated with Lipofectamine 2000 transfection reagent (both from Invitrogen, San Diego, CA, USA) (7). Transfected cells were selected with G418 $(100 \mu \mathrm{g} / \mathrm{ml}$ for BGC-SP and SGC-SP clones) for 14 days before the isolation of individual clones.

Western blotting. Total cell lysates were prepared and analyzed by WB as previously described (6,7). Protein expression in cell lysates were normalized by the housekeeping protein GAPDH.

Cell proliferation assay. Cell proliferation was determined by an MTS assay (Promega, Madison, WI, USA). Briefly, cells were incubated per well in $100 \mu \mathrm{l}$ media using 96-well plates and incubated. At different time points, $20 \mu \mathrm{l}$ MTS was added to the cells. Absorbance values at $490 \mathrm{~nm}$ were measured.

Cell cycle analysis by FCM analysis. The allocation of cells in the cell cycle phases was determined using FCM analysis of DNA content. Cells were collected and fixed in $70 \%$ ethanol, and stained with propidium iodide for $30 \mathrm{~min}$ and analyzed for DNA content by using FCM (Becton-Dickinson, San Jose, CA, USA).

Matrigel colony assay. GC cells were infected with either SPARC cDNA or empty vectors and parental cells were grown on Matrigel-coated $(200 \mu \mathrm{l}$ at $11.5 \mathrm{mg} / \mathrm{ml}) 14 \mathrm{~mm}$ microwell for 14 days, and then colonies were monitored using phase-contrast light microscope. Five fields per sample were captured using a digital camera. The number of the colonies was assessed using ImageJ software. This experiment was repeated thrice. 


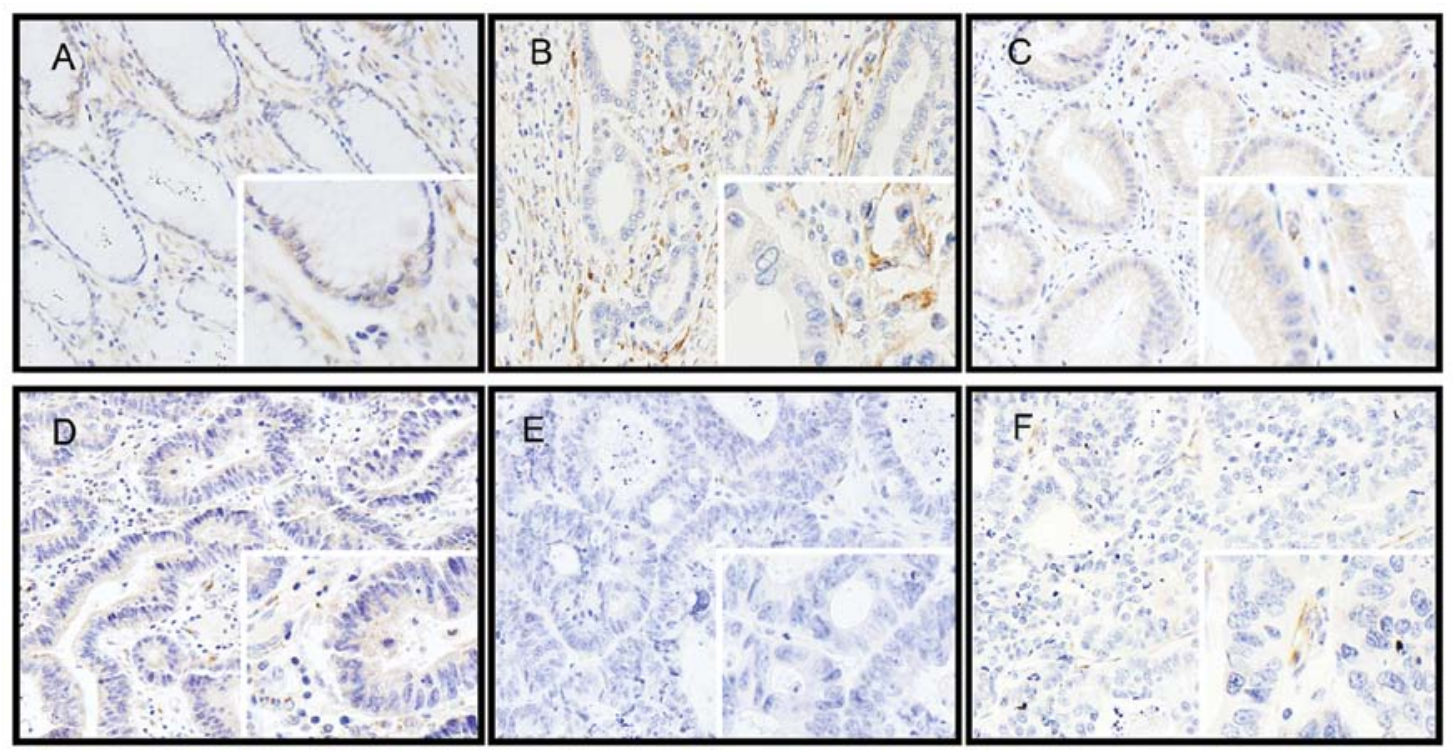

Figure 1. The immunostaining of SPARC in normal gastric mucosa and gastric cancer. (A) Normal epithelial and stromal cells expressed faint level of SPARC in cytoplasm. (B and C) Immunostaining of SPARC was weak in well differentiated intestinal gastric cancer. However, the stromal cells within and surrounding the tumor showed staining of variable intensity. (D and E) Immunostaining of SPARC was faint or absent in moderate differentiated intestinal gastric cancer. (F) Immunostaining of SPARC was absent in poor differentiated diffuse gastric cancer cells and faint in stromal cells within the tumor. SPARC, secreted protein acidic and rich in cysteine.

CD44 FCM. For FCM analysis, cells grown for $48 \mathrm{~h}$ were washed and detached with $1.25 \mathrm{mM}$ EDTA in PBS. After washing with ice-cold medium, cells were incubated with a monoclonal CD44 antibody for $30 \mathrm{~min}$, washed twice with PBS $0.1 \%$ BSA. After washings, samples were incubated for $30 \mathrm{~min}$ with a secondary goat FITC-conjugated anti-rabbit antibody at room temperature. Cells were washed, resuspended in PBS and subjected to FCM. This experiment was repeated thrice.

Cell invasion and migration assays. To detect cell invasion, a 24-well Transwell plate consisting of Boyden chambers with pre-coated Matrigel membrane filter was used (pore size, $8 \mu \mathrm{m}$ ). RPMI $(500 \mu \mathrm{l})$ containing 10\% FBS was added to the lower chamber and $1 \times 10^{4}$ cells in $200 \mu \mathrm{l}$ of serum-free RPMI were plated into the upper chamber. The chamber was incubated for $36 \mathrm{~h}$ at $37^{\circ} \mathrm{C}$. Then, the upper surface of the filters was removed and the invaded cells were fixed with $4 \%$ paraformaldehyde and stained with $0.1 \%$ crystal violet. The number of invaded cells was quantified by counting five random fields per filter. To detect cell migration, a 24-well Transwell plate (Matrigeluncoated) was used and the procedure was the same as for the cell invasion assay. Both assays were performed in triplicate.

Nude mice models. The studies were performed in accordance with a protocol approved by the Animal Care and Use Committee of Peking University (ethical application approval no. J201155). The athymic nude mice were randomized to different groups ( $n=6 /$ group). Lung metastasis mouse models were established by tail vein injection with human BGC-P, BGC-EV, BGC-SP cells ( $5 \times 10^{5}$ cells/mouse). Mouse models were monitored for 30 days, which was the termination point of the experiment. In order to assess the metastases of cancer cells, lung samples of mice were fixed in formalin at $4^{\circ} \mathrm{C}$. Lung samples were then embedded in paraffin. Five sections from different depths of lung samples were analyzed, and the distance of adjacent depths was $100 \mu \mathrm{m}$. The slides were deparaffinized and rehydrated as described above. Sections were then stained with hematoxylin-eosin (H\&E). Cell and colony numbers of lung metastases were counted under a microscope.

Statistical analysis. Table 1 presents a summary of the analyzed medical parameters in the form of frequency distributions for discrete parameters, respectively. All SPARC expression levels were analyzed by Pearson's Chi-square or Fisher's exact tests. Other data are expressed as the means \pm SD. Statistical analysis was performed using one-way ANOVA followed by Dunnett's multiple comparison or a Student's t-test. $\mathrm{P}<0.05$ was considered to indicate a statistically significant difference. All tests were carried out with SPSS 13.0.

\section{Results}

SPARC expression is negatively correlated with clinicopathological factors of GCs. We tested SPARC expression using IHC in normal gastric mucosa and in cancer tissues. In normal gastric tissues, SPARC was expressed faintly in the cytoplasm of normal mucosal epithelial and stromal cells. In GC tissues, immunostaining was weak or absent in cancer cells. However, immunoreaction was variable and common in the cells of the desmoplastic stroma surrounding the cancer cells (Fig. 1). No statistically significant correlations were observed between SPARC expression and gender, age at diagnosis, tumor location, tumor size, tumor differentiation or metastases to distant tissues. Decreased staining intensity of SPARC was found with advanced TNM staging $(\mathrm{P}=0.002)$, degree of stomach wall invasion ( $\mathrm{T}$ staging, $\mathrm{P}=0.013)$, lymph node metastasis ( $\mathrm{N}$ staging, $\mathrm{P}=0.009$ ). We found that SPARC expression significantly correlated with tumor type, favoring the intestinal type 
Table I. Patient clinicopathological factors and SPARC expression in gastric cancer tissues.

\begin{tabular}{|c|c|c|c|c|}
\hline Parameters & $\begin{array}{c}\text { No. }(\%) \\
\mathrm{n}=65\end{array}$ & $\begin{array}{c}\text { SPARC IHC } \\
\text { High score }(\%) \\
\mathrm{n}=23\end{array}$ & $\begin{array}{c}\text { SPARC IHC } \\
\text { Low score }(\%) \\
\mathrm{n}=42\end{array}$ & P-value \\
\hline Gender & & & & $0.831^{\mathrm{a}}$ \\
\hline Male & $47(72.31)$ & $17(36.2)$ & $30(63.8)$ & \\
\hline Female & $18(27.69)$ & $6(33.3)$ & $12(66.7)$ & \\
\hline Age (years) & & & & $0.384^{\mathrm{a}}$ \\
\hline$<65$ & $32(49.23)$ & $13(40.4)$ & $19(59.4)$ & \\
\hline$\geq 65$ & $33(50.77)$ & $10(30.3)$ & $23(69.7)$ & \\
\hline TNM stage & & & & $0.002^{\mathrm{a}}$ \\
\hline $\mathrm{I} / \mathrm{II}$ & $31(47.69)$ & $17(54.8)$ & $14(45.2)$ & \\
\hline III/IV & $34(52.31)$ & $6(17.6)$ & $28(82.4)$ & \\
\hline Histological type & & & & $0.036^{\mathrm{b}}$ \\
\hline Intestinal & $49(75.38)$ & $21(63.3)$ & $28(36.7)$ & \\
\hline Diffuse & $16(24.62)$ & $2(31.3)$ & $14(68.8)$ & \\
\hline Diameter & & & & $0.672^{\mathrm{a}}$ \\
\hline$<5 \mathrm{~cm}$ & $26(40.0)$ & $10(38.5)$ & $16(61.5)$ & \\
\hline$\geq 5 \mathrm{~cm}$ & $39(60.0)$ & $13(33.3)$ & $26(66.7)$ & \\
\hline Differentiation & & & & $0.078^{\mathrm{a}}$ \\
\hline Well/moderate & $30(46.15)$ & 14 (46.7) & $16(53.3)$ & \\
\hline Poor & $35(53.85)$ & $9(25.7)$ & $26(74.3)$ & \\
\hline Location & & & & $0.590^{\mathrm{a}}$ \\
\hline Upper third & $12(18.46)$ & $3(25.0)$ & $9(75.0)$ & \\
\hline Middle third & $42(64.62)$ & $15(35.7)$ & $27(64.3)$ & \\
\hline Lower third & $11(16.92)$ & $5(45.5)$ & $6(54.5)$ & \\
\hline Tumor infiltration & & & & $0.013^{\mathrm{a}}$ \\
\hline $\mathrm{T} 1 / \mathrm{T} 2$ & $10(15.38)$ & $7(70.0)$ & $3(30.0)$ & \\
\hline $\mathrm{T} 3 / \mathrm{T} 4$ & $55(84.62)$ & $16(29.1)$ & $39(70.9)$ & \\
\hline Local lymph node metastasis & & & & $0.009^{\mathrm{a}}$ \\
\hline No & $16(24.62)$ & $10(62.5)$ & $6(37.5)$ & \\
\hline N1-N3 & $49(75.38)$ & $13(26.5)$ & $36(73.5)$ & \\
\hline Distant metastasis & & & & $0.941^{\mathrm{a}}$ \\
\hline M0 & $54(83.08)$ & $19(23.8)$ & $35(76.2)$ & \\
\hline M1 & $11(16.92)$ & $4(9.1)$ & $7(90.9)$ & \\
\hline
\end{tabular}

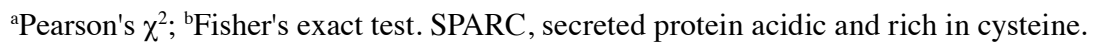

but not the diffuse type of GC. The immunoreactivity in the intestinal cancer was high in $21(42.86 \%)$ cases, and low in 28 $(57.14 \%)$ cases. Diffuse GC cells showed a high reaction in 2 $(12.5 \%)$ and a low reaction in $14(87.5 \%)$ cases; the difference was significant $(\mathrm{P}=0.036$, Table $\mathrm{I})$.

Endogenous SPARC overexpression suppresses the growth rate, and the deactivation of VEGFR2 is involved in the reduction of growth rate and cycle progression. When BGC-SP and SGC-SP cells were transduced with pcDNA3.1 plasmid carrying the cDNAs encoding SPARC, a $>20$-fold increase in the abundance of the protein was observed (Fig. 2A). MTS assays showed that SPARC overexpression reduced the growth rate of GC cells significantly $(\mathrm{P}<0.05$; Fig. $2 \mathrm{~A})$. In our previous study, we showed that endogenous SPARC overexpression suppressed the expression of VEGF and deactivated the ERK1/2 signaling pathway (7). The expression of cyclin-D1 and $\mathrm{p} 21$ was tested in the present study. The results of WB showed that the cyclin-D1 expression was reduced while p21 expression was increased (Fig. 2A). It is believed that VEGF plays an important role in proliferative activity. In order to exclude the effect of reduced endogenous VEGF expression induced by SPARC overexpression and effects from other growth factors, exogenous recombination human VEGF was added in serum-free media of cell lines; heparin was used as control. The cell lines were cultured for $48 \mathrm{~h}$; the proliferation and cycle progression of cell lines were tested and the phosphoVEGFR2 and ERK1/2 expressions were tested. 
A

C

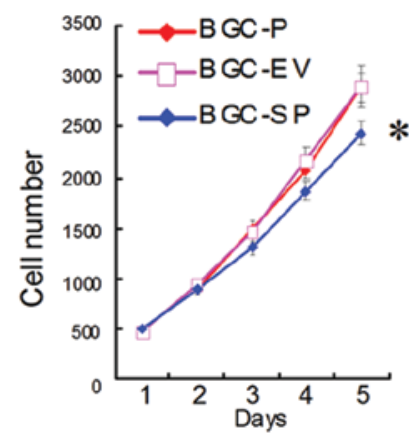

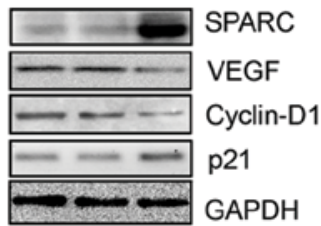

i.

创
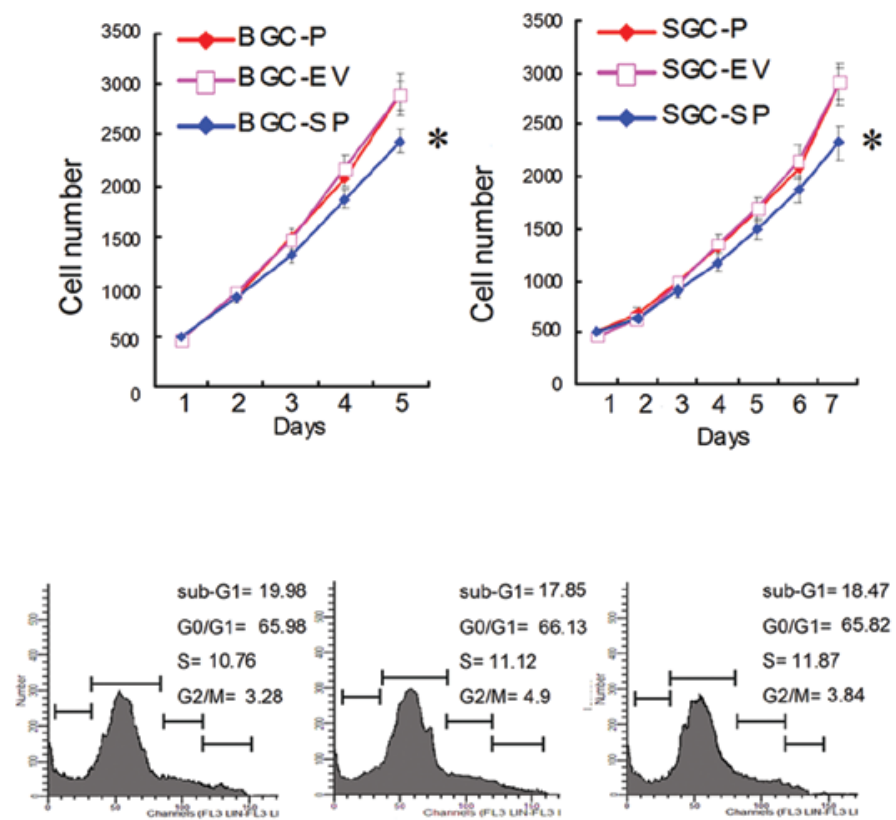

BGC-P

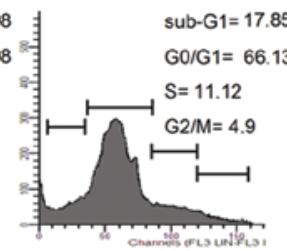

BGC-EV

+ Heparin

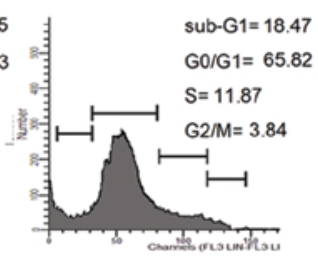
BGC-SP

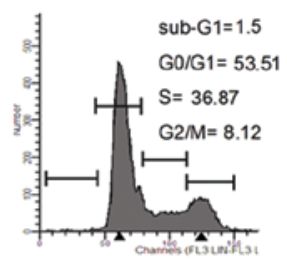

BGC-P

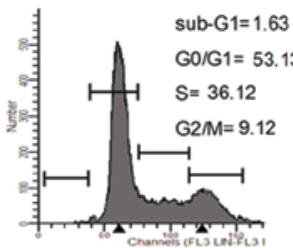

BGC-EV

+ VEGF
B
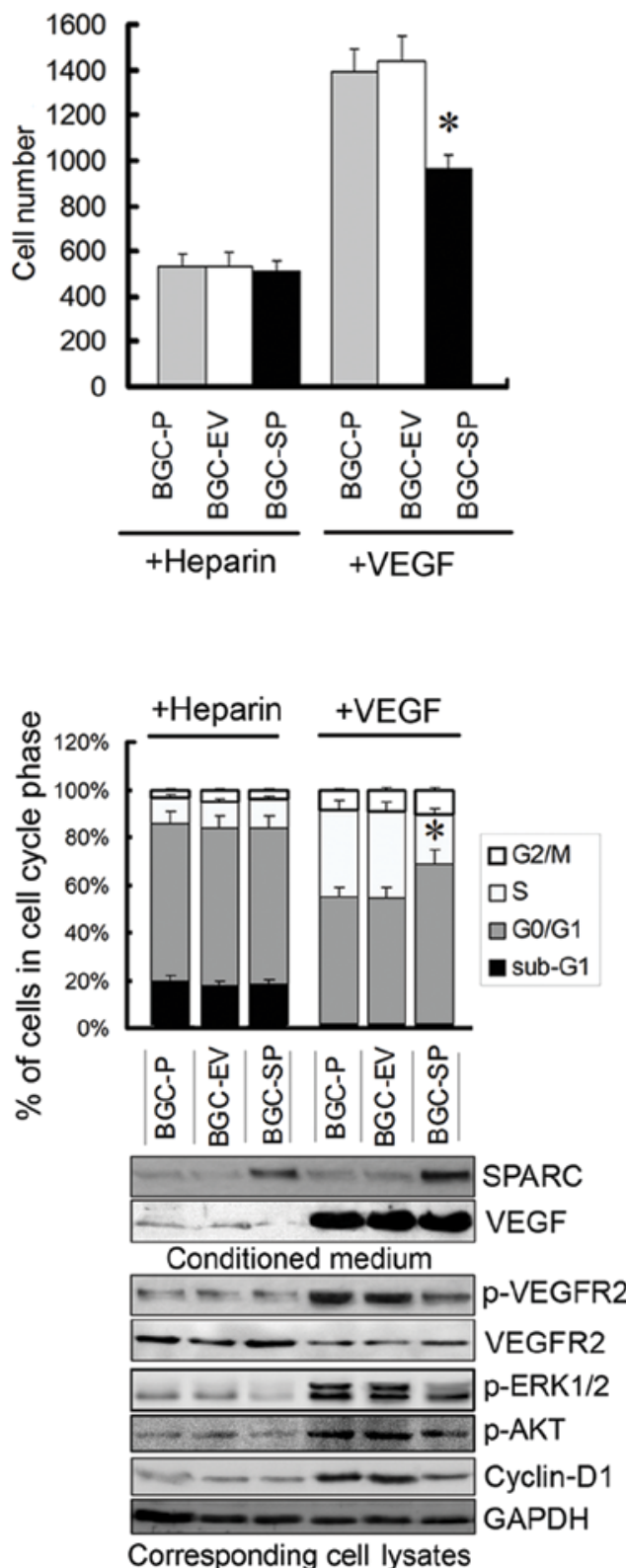

Corresponding cell lysates

Figure 2. SPARC overexpression suppresses the growth rate and arrests the cell cycle progression of gastric cancer cells induced by VEGF. (A) SPARC expression of BGC-823 and SGC-7901 cells transfected with SPARC cDNA (BGC-SP and SGC-SP cells) was increased significantly compared with empty vector-transfected (BGC-EV and SGC-EV cells) and parental cell lines (BGC-P and SGC-P cells). By contrast, the expression of VEGF and cyclin-D1 was reduced and p21 was increased in BGC-SP and SGC-SP cells. The results of proliferation assays are shown as cell numbers, and the data are expressed as means \pm SD of quadruplicate determinations from three separate experiments. SPARC overexpression reduced the growth rate of gastric cancer cells ("P $<0.05)$. (B) BGC-SP, BGC-EV and BGC-P cells were cultured for $48 \mathrm{~h}$ in serum-free medium in the presence of heparin $(1 \mathrm{mg} / \mathrm{ml})$ or $5 \mathrm{nM}$ VEGF. The proliferation of cells was determined by MTS assay. The results are shown as cell numbers, and the data are expressed as means \pm SD of quadruplicate determinations from three separate experiments. SPARC overexpression inhibited the growth rate induced by VEGF significantly ( $\mathrm{P}<0.05)$. (C) The cellular DNA content was measured by flow cytometry (FCM) to determine cell cycle progression. The columns are shown as \% of cells in cell cycle phases, and the data are expressed as means \pm SD of three separate experiments. FCM assay showed that SPARC overexpression arrested the cell cycle in G0/G1 phase ("P<0.05). The activation of VEGFR2, ERK1/2, AKT and the expression of cyclin-D1 were declined in BGC-SP cells compared with empty vector-transfected and parental cells. SPARC, secreted protein acidic and rich in cysteine.

By contrast, the proliferation of BGC-SP cells was reduced by $30 \%$ when VEGF concentration in serum-free media was equal to that of empty vector-transduced or parental cell lines (P<0.05; Fig. 2B). To determine whether endogenous SPARC expression results in cell cycle changes, the cellular DNA content was measured by FCM. When cell lines were treated by exogenous VEGF, the percentage of BGC-SP cells in the G0/ G1 phase was significantly increased when compared with the 

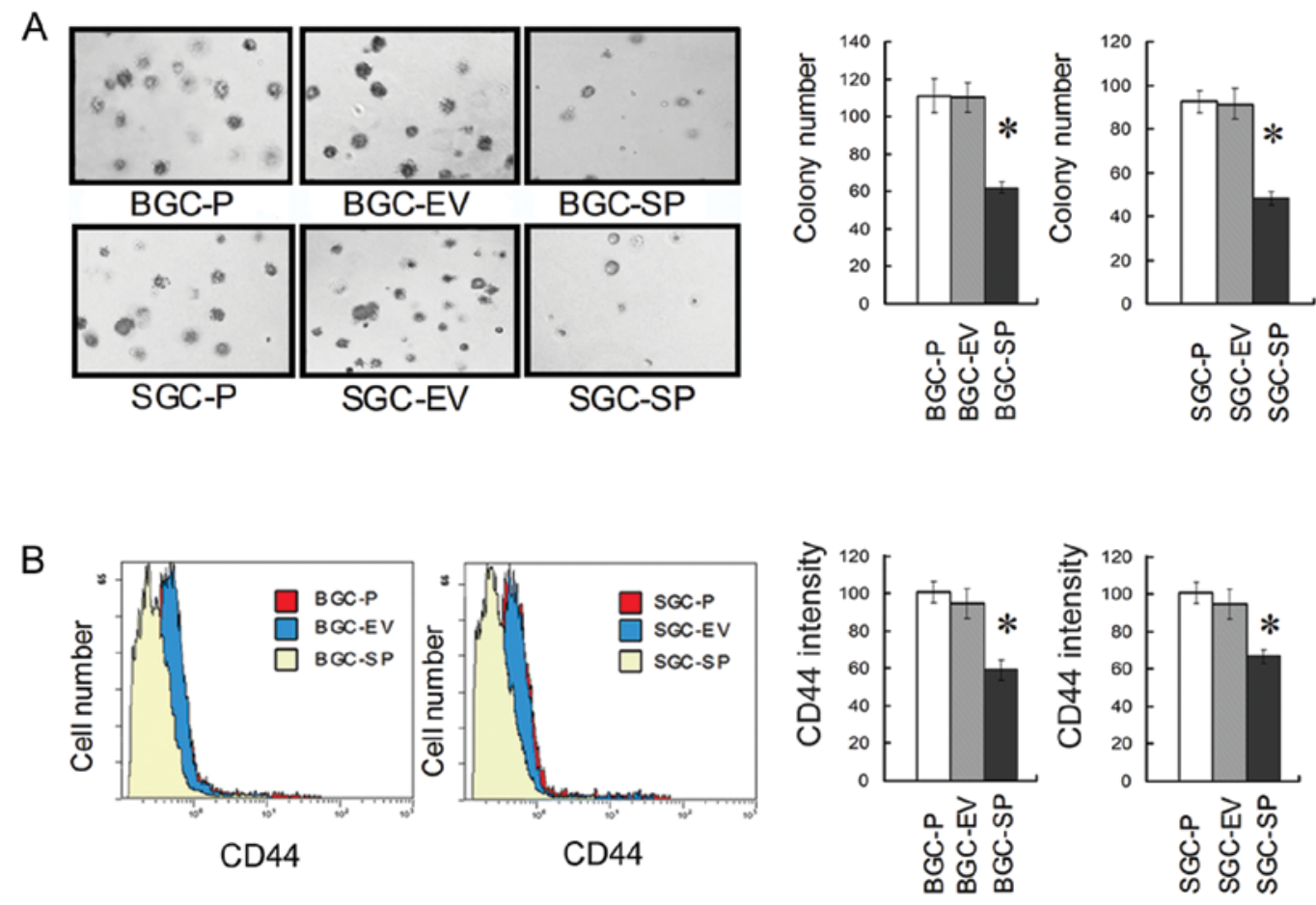

Figure 3. Reduction of clonogenic capacity of gastric cancer cells is regulated by decreased CD44 expression driven by SPARC overexpression. (A) Images of the spheroids from gastric cancer cells at day 14 were captured under phase-contrast light microscope. The results are shown as colony numbers, and the data are expressed as means \pm SD of quadruplicate determinations from three separate experiments. Forced SPARC expression led to declined clonogenicity of gastric cancer cells ( $\mathrm{P}<0.05)$. (B) Flow cytometry assay was used to determine the expression of CD44 of gastric cancer cells. The results are shown as mean fluorescence intensity of CD44 (in \%) of respective parental cells and are means \pm SD of three separate experiments. The CD44 expression of BGC-SP and SGC-SP cells decreased by $30-40 \%$ compared with parental cells, respectively $\left({ }^{*} \mathrm{P}<0.05\right)$. SPARC, secreted protein acidic and rich in cysteine.

control cell lines $(\mathrm{P}<0.05$; Fig. 2C). By contrast, the percentage of BGC-SP cells in the S-phase was significantly decreased $(\mathrm{P}<0.05$; Fig. $2 \mathrm{C})$. These data demonstrated that SPARC overexpression induced a significant arrest of cell cycle progression in the G0/G1 phase when the same concentration of VEGF was used in the media. The proliferation and cycle progression of the BGC-SP cell line were not affected significantly when heparin was used as control. The activation of VEGFR2, ERK1/2, AKT and expression of cyclin-D1 was decreased in cells overexpressing SPARC (Fig. 2C). Presumably, SPARC interfered with the binding between VEGF and VEGFR2, therefore, the activation of VEGFR2 and downstream ERK1/2 was inhibited.

Endogenous SPARC overexpression suppresses clonogenicity of $G C$ cells by decreasing CD44. Clonogenic assay revealed that SPARC overexpression reduced the clonogenicity of GC cells significantly $(\mathrm{P}<0.05$; Fig. $3 \mathrm{~A})$. The FCM assay showed an $\sim 30-40 \%$ reduction in CD44 cell surface expression level in SPARC-overexpressing cell lines when compared to empty vector-transduced or parental cell lines, respectively $(\mathrm{P}<0.05$; Fig. 3B).

Endogenous SPARC overexpression suppresses the invasion and migration of $G C$ cells by decreasing the expression of $N$-cadherin, MMP-7, MMP-9, Spl and p-ERK1/2 expression. To determine whether SPARC overexpression affects migration and invasion of GCs, cell migration and invasion assays were performed using Boyden chambers. We measured the capacity of GC cells to invade through the chamber membrane coated or not coated with Matrigel. At $36 \mathrm{~h}$, there was a 40-50\% decrease in migration of SPARC cDNA-transfected GC cells, compared with empty vector-transfected and parental cells, respectively ( $\mathrm{P}<0.05$; Fig. 4). In addition, there was a $40-50 \%$ decrease in invasion of SPARC cDNA-transfected GC cells, compared with empty vector-transfected and parental cells, respectively ( $\mathrm{P}<0.05$; Fig. 4). Collectively, these results clearly indicated that SPARC overexpression led to the inhibition of invasion and migration significantly, in both BGC-823 and SGC-7901 cells, respectively. By contrast, WB revealed that the expression of MMP-7, MMP-9, N-cadherin, Sp1 and p-ERK1/2 was significantly inhibited in BGC-SP and SGC-SP cells compared with the control cell lines. However, the expression of Snail and E-cadherin was not significantly affected (Fig. 4).

In vivo assay: lung metastases of GC are suppressed by endogenous SPARC overexpression. In order to determine the capacity of GC cells to form metastases in the lung, nude mice models were established by tail vein injection with human BGC-P, BGC-EV, BGC-SP cells ( $5 \times 10^{5}$ cells/mouse). The lung samples were fixed in formalin and embedded in the paraffin within 30 days. Five sections from different depths of lung samples were stained and analyzed. The statistical analysis revealed that there was no significant difference in lung metastases between BGC-P cells (metastatic cell no. 2508.56 \pm 623.69 ; colony no. $85.23 \pm 16.92)$ and BGC-EV cells (metastatic cell no. 2714.21 \pm 646.92 ; colony no. $88.72 \pm 21.36$ ). Metastatic cell number and colony number were decreased significantly in BGC-SP cells (metastatic cell no. $1485.68 \pm 243.19$; colony no. $41.73 \pm 7.22$, P $<0.05$; Fig. 5). 

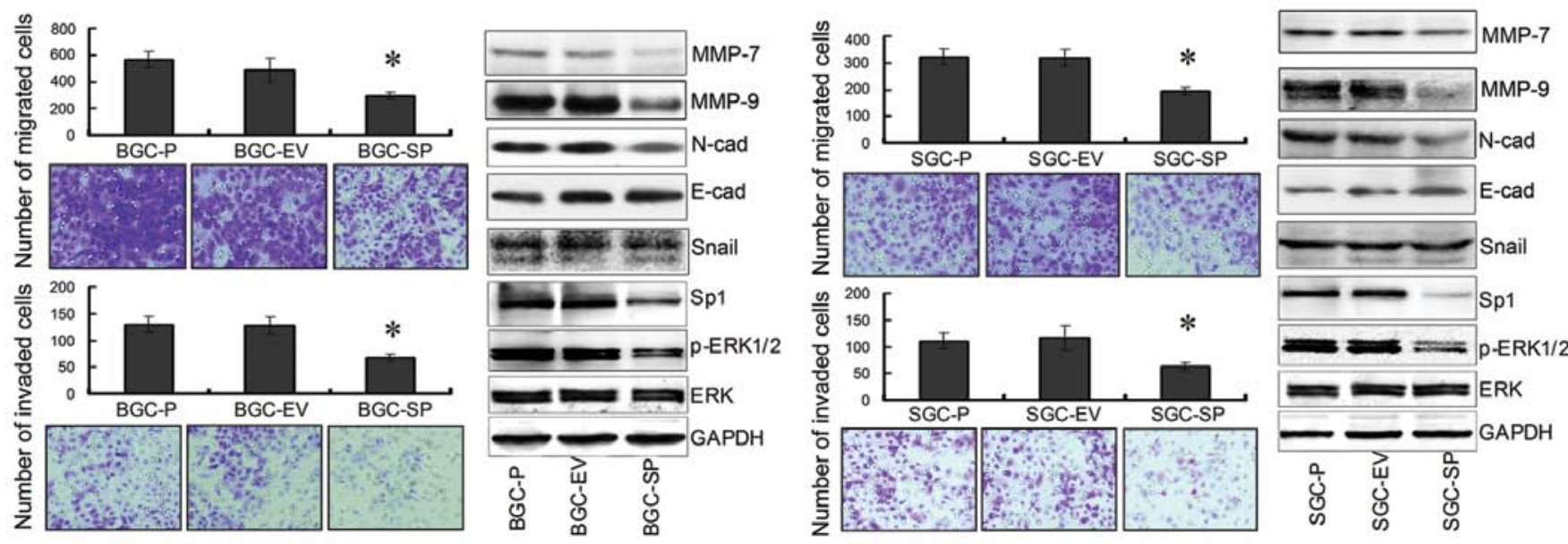

Figure 4. Enforced expression of SPARC suppresses the migration and invasion of gastric cancer cells. The number of cells that invaded and migrated to the lower side of the Boyden chamber was determined as described in the Materials and methods section for SPARC cDNA-transfected gastric cells, empty vectortransfected and parental cells, respectively. The results are expressed as means $\pm \mathrm{SD}$ of invaded or migrated cells within $36 \mathrm{~h}$ of three separate experiments. SPARC overexpression suppressed the number of invaded and migrated cells significantly ( $\mathrm{P}<0.05$ ). By contrast, western blotting revealed that the expression of MMP-7, MMP-9, N-cadherin, Sp1 and p-ERK1/2 expression was significantly inhibited in BGC-SP and SGC-SP cells compared with the control cell lines. SPARC, secreted protein acidic and rich in cysteine.

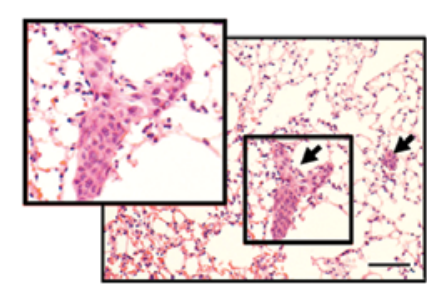

BGC-P

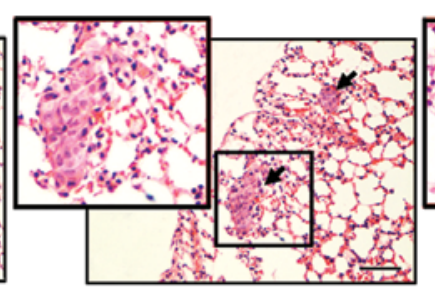

BGC-EV

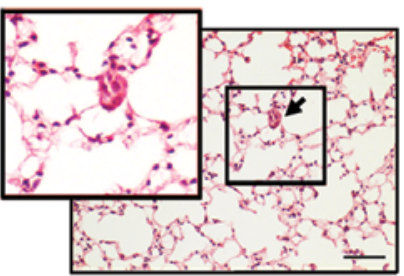

BGC-SP
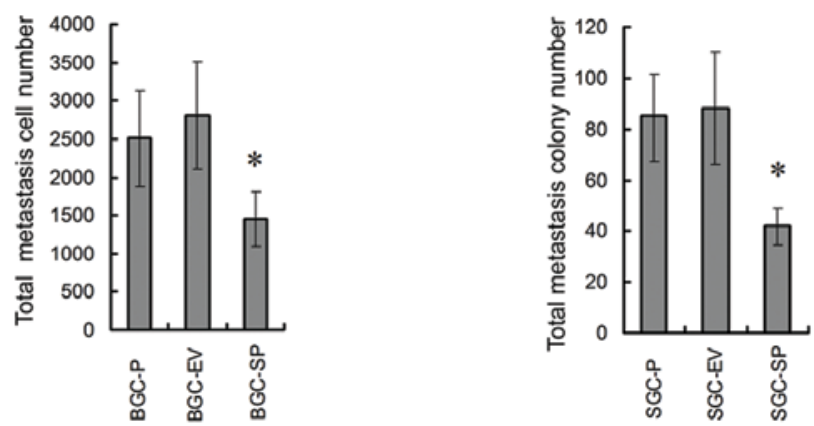

Figure 5. In vivo assay; lung metastases of gastric cancer are suppressed by endogenous SPARC overexpression. Mouse models of lung metastasis were established by tail vein injection with human BGC-P, BGC-EV, BGC-SP cells $\left(5 \times 10^{5}\right.$ cells/mouse). The lung samples were fixed in formalin and embedded in the paraffin at day 30. Five sections from different depths of lung samples were stained by H\&E and analyzed. Arrows point to the colonies of metastases. The columns are shown as total metastatic cell and colony numbers of each mouse from six mice in each group (means \pm SD). " $\mathrm{P}<0.05$, significant difference from the parental group. SPARC, secreted protein acidic and rich in cysteine.

\section{Discussion}

SPARC is known to be involved in diverse biological processes, including collagen fibrinogenesis, wound repair, apoptosis and in reducing proliferation, migration and angiogenesis $(3,15)$. High levels of SPARC expression negatively correlate with the overall survival and disease-free survival of patients with breast cancer (16). Our previous study showed that exogenous SPARC significantly decreased the growth and migration of pancreatic cancer cells $(6,17)$. The present study, as well as other studies, showed that SPARC inhibited the angiogenesis and VEGF expression of different types of cancer (4,7,18-20).

To explore if SPARC promotes or suppresses the development and progression of GC, in the present study, we tested the expression of SPARC in GC tissues and non-cancerous normal tissues from patients. Our IHC study showed that SPARC expression was faint in normal epithelial and stromal cells. However, in most GC tissues, SPARC was almost unde- 
tectable in epithelial cancer cells, whereas variable SPARC expression was observed in the cytoplasm of stromal cells surrounding the GC; these results are consistent with results of other studies (13,21-23). Statistical analysis showed that SPARC expression was negatively correlated with clinicopathological parameters including TNM stage, tumor infiltration and lymph node metastasis. SPARC expression did not show a statistical correlation with tumor differentiation and metastasis in distant sites. This may be due to the limited number of patients included in the experiment. We found that SPARC expression highly significantly correlated with tumor type, favoring the intestinal type but not the diffuse type of GC. It is believed that, in the diffuse type of GC, a reduced expression of SPARC in stromal cells from GCs may support a decohesive phenotype (13). It is widely accepted that SPARC binds to several types of collagen, which are the major structural proteins of the extracellular matrix (ECM) produced by host stromal cells in response to the tumor cells. It is proposed that SPARC inhibits proliferation of tumors in primary and metastatic sites at least in part by increasing the collagen content and mechanical stiffness of the fibers surrounding the tumor, thus restricting the growth of the tumor (24). The results of Wang et al (14) were similar to ours and demonstrated that SPARC may be a potential tumor suppressor in GC.

To address this possibility, we established BGC-823 and SGC-7901 cell lines which overexpressed SPARC. Our in vitro assays revealed that endogenous SPARC overexpression significantly inhibited the proliferation of GC. Our previous study demonstrated that endogenous SPARC suppressed the proliferation and expression of VEGF at the same time. It is believed that VEGF is a considerable cause of promoting the growth of tumors. In order to further investigate the mechanism of deproliferative function of SPARC, $5 \mathrm{nM}$ exogenous rhVEGF was used in the media of BGC-P, BGC-EV and BGC-SP cells were identical. MTS and FCM showed that, in the media with the same concentrations of VEGF, the proliferation and cell cycle progression of SPARC overexpressing cells were inhibited. Furthermore, the activation of VEGFR2, ERK1/2, AKT of BGC-SP were suppressed, which meant SPARC suppressed the proliferation at least in part by prohibiting the activation of VEGFR2 induced by VEGF.

In addition to SPARC overexpression suppressing proliferation of GC, our in vitro assays revealed that endogenous SPARC overexpression significantly inhibited the clonogenicity of GC on Matrigel. The declonogenic effect of SPARC suggests that this factor may play a role in a stem-like population of GC cells. CD44 was identified as a potential biomarker of stem cells in GC. It is believed that the clonogenic capacity of cells expressing high level of CD44 is much stronger than cells expressing low level of CD44 (25). The CD44 expression was tested by FCM. By contrast, the CD44 expression was reduced significantly in SPARC-overexpressing GC cells.

In our clinical experiments, absence of SPARC expression in GC tissues correlated with advanced stages of stomach wall invasion, lymph node metastasis. According to these results from clinical experiments, we hypothesized that SPARC played a key role in invasion and migration of GC. To address this possibility, Transwell assays were arranged. By contrast, the invasion and migration were suppressed in SPARCoverexpressing GC cells. One of the key processes providing cancer cells with the capacity to migrate, invade and metastasize is their ability to undergo an EMT. EMT is characterized by loss of intercellular adhesion (E-cadherin to N-cadherin switch), upregulation of Snail and MMPs. The western blotting showed that endogenous SPARC inhibited the expressions of MMP-7, MMP-9, N-cadherin, Sp1 and the activation of ERK1/2 significantly, but did not affect the expression of Snail and E-cadherin significantly. MMPs were thought to predominantly degrade specific components of the ECM, thereby providing new substrates facilitating migration and invasion. $\mathrm{N}$-cadherin is an important biomarker of EMT. In N-cadherintransfected breast cancer cells, $\mathrm{N}$-cadherin promotes motility and invasion, but the reduction in the expression of E-cadherin does not necessarily correlate with either of these two (26). This finding indicated that $\mathrm{N}$-cadherin, functioning as adhesion molecules, may be more important than E-cadherin for metastasis and invasion. It is believed that Sp1 is one of the most important transcription factors which promotes the invasion and metastasis of different cancers.

In vivo assay showed that metastases in the lungs of nude mice were suppressed by SPARC overexpression. This result demonstrated that SPARC overexpression suppressed the proliferation, clonogenicity and invasion of GC cells in nude mice.

In summary, our results illustrated that SPARC expression in GC tissues was negatively correlated with clinicopathological parameters of patients. SPARC inhibited the proliferation via decoction of VEGFR2, ERK, AKT and SPARC inhibited invasion of GC via reduced expression of MMP-7, MMP-9, $\mathrm{N}$-cadherin and Sp1. We conclude that SPARC expression probably suppresses the malignancy of GC, and the exploration aimed to regulate SPARC expression may become a beneficial approach to improve GC treatment.

\section{Acknowledgements}

This study was financially supported by grants from the National Natural Science Foundation of China (no. 30901417/H1617).

\section{References}

1. Jemal A, Bray F, Center MM, Ferlay J, Ward E and Forman D: Global cancer statistics. CA Cancer J Clin 61: 69-90, 2011.

2. Ferlay J, Shin HR, Bray F, Forman D, Mathers C and Parkin DM: Estimates of worldwide burden of cancer in 2008: GLOBOCAN 2008. Int J Cancer 127: 2893-2917, 2010.

3. Tai IT and Tang MJ: SPARC in cancer biology: its role in cancer progression and potential for therapy. Drug Resist Updat 11: 231-246, 2008

4. Liang JF, Wang HK, Xiao H, et al: Relationship and prognostic significance of SPARC and VEGF protein expression in colon cancer. J Exp Clin Cancer Res 29: 71, 2010.

5. Atorrasagasti C, Malvicini M, Aquino JB, et al: Overexpression of SPARC obliterates the in vivo tumorigenicity of human hepatocellular carcinoma cells. Int J Cancer 126: 2726-2740, 2010.

6. Chen G, Tian X, Liu Z, et al: Inhibition of endogenous SPARC enhances pancreatic cancer cell growth: modulation by FGFR1-III isoform expression. Br J Cancer 102: 188-195, 2010.

7. Zhang JL, Chen GW, Liu YC, et al: Secreted protein acidic and rich in cysteine (SPARC) suppresses angiogenesis by downregulating the expression of VEGF and MMP-7 in gastric cancer. PLoS One 7: e44618, 2012.

8. Chlenski A and Cohn SL: Modulation of matrix remodeling by SPARC in neoplastic progression. Semin Cell Dev Biol 21: $55-65,2010$.

9. Nagaraju GP and El-Rayes BF: SPARC and DNA methylation: possible diagnostic and therapeutic implications in gastrointestinal cancers. Cancer Lett 328: 10-17, 2013. 
10. Hasselaar P and Sage EH: SPARC antagonizes the effect of basic fibroblast growth factor on the migration of bovine aortic endothelial cells. J Cell Biochem 49: 272-283, 1992.

11. Funk SE and Sage EH: The $\mathrm{Ca}^{2+}$-binding glycoprotein SPARC modulates cell cycle progression in bovine aortic endothelial cells. Proc Natl Acad Sci USA 88: 2648-2652, 1991.

12. Said N and Motamed K: Absence of host-secreted protein acidic and rich in cysteine (SPARC) augments peritoneal ovarian carcinomatosis. Am J Pathol 167: 1739-1752, 2005.

13. Franke K, Carl-McGrath S, Röhl FW, et al: Differential expression of SPARC in intestinal-type gastric cancer correlates with tumor progression and nodal spread. Transl Oncol 2: 310-320, 2009.

14. Wang L, Yang M, Shan L, et al: The role of SPARC protein expression in the progress of gastric cancer. Pathol Oncol Res 18: 697-702, 2012.

15. Arnold SA and Brekken RA: SPARC: a matricellular regulator of tumorigenesis. J Cell Commun Signal 3: 255-273, 2009.

16. Nagai MA, Gerhard R, Fregnani JH, et al: Prognostic value of NDRG1 and SPARC protein expression in breast cancer patients. Breast Cancer Res Treat 126: 1-14, 2011.

17. Liu YP and Hsiao M: Exercise-induced SPARC prevents tumorigenesis of colon cancer. Gut 62: 810-811, 2013.

18. Yunker CK, Golembieski W, Lemke N, et al: SPARC-induced increase in glioma matrix and decrease in vascularity are associated with reduced VEGF expression and secretion. Int $\mathrm{J}$ Cancer 122: 2735-2743, 2008.

19. Bhoopathi P, Chetty C, Gujrati M, Dinh DH, Rao JS and Lakka SS: The role of MMP-9 in the anti-angiogenic effect of secreted protein acidic and rich in cysteine. Br J Cancer 102: 530-540, 2010.
20. Lau CP, Poon RT, Cheung ST, Yu WC and Fan ST: SPARC and Hevin expression correlate with tumour angiogenesis in hepatocellular carcinoma. J Pathol 210: 459-468, 2006.

21. Takeno A, Takemasa I, Doki Y, et al: Integrative approach for differentially overexpressed genes in gastric cancer by combining large-scale gene expression profiling and network analysis. $\mathrm{Br} \mathrm{J}$ Cancer 99: 1307-1315, 2008.

22. Junnila S, Kokkola A, Mizuguchi T, et al: Gene expression analysis identifies over-expression of $C X C L 1, S P A R C, S P P 1$, and SULF1 in gastric cancer. Genes Chromosomes Cancer 49: 28-39, 2010.

23. Wang CS, Lin KH, Chen SL, Chan YF and Hsueh S: Overexpression of SPARC gene in human gastric carcinoma and its clinic-pathologic significance. Br J Cancer 91: 1924-1930, 2004.

24. Chong HC, Tan CK, Huang RL and Tan NS: Matricellular proteins: a sticky affair with cancers. J Oncol 2012: 351089, 2012.

25. Takaishi S, Okumura T, Tu S, et al: Identification of gastric cancer stem cells using the cell surface marker CD44. Stem Cells 27: 1006-1020, 2009.

26. Nieman MT, Prudoff RS, Johnson KR and Wheelock MJ: $\mathrm{N}$-cadherin promotes motility in human breast cancer cells regardless of their E-cadherin expression. J Cell Biol 147: 631-644, 1999 